\title{
Topological clusters in SU(2) gluodynamics at finite temperature and the evidence for KvB calorons
}

\section{Ernst-Michael llgenfritz*}

Institut für Physik, Humboldt Universität zu Berlin, 12489 Berlin, Germany

E-mail: ilgenfri@physik.hu-berlin.de

\section{Philipp Gerhold and Michael Müller-Preussker}

Institut für Physik, Humboldt Universität zu Berlin, 12489 Berlin, Germany

E-mail: gerhold@physik.hu-berlin.de, mmp@physik.hu-berlin.de

\section{Boris V. Martemyanov and Alexander I. Veselov}

ITEP, Bolshaya Cheremushkinskaya 25, 117259 Moscow, Russia

E-mail: martemja@itep.ru, veselov@itep.ru

\begin{abstract}
We report on our search for Kraan-van Baal calorons in finite temperature SU(2) lattice ensembles. We also discuss recent progress made in developing a caloron-anticaloron gas model decribing confinement and deconfinement in the context of trivial and non-trivial holonomy.
\end{abstract}

XXIIIrd International Symposium on Lattice Field Theory

25-30 July 2005

Trinity College, Dublin, Ireland

${ }^{*}$ Speaker. 


\section{Introduction}

With the help of overlap fermions, we understand better today how the topological charge is distributed in the vacuum, seen without or with an UV filter [1,2,3]. Still, there is a quest for a picture in terms of semiclassical gluonic field excitations which can be held responsible for the nonperturbative properties of QCD. Indeed, a locally (anti)selfdual background becomes visible under moderate smearing of a lattice gauge fields taken from the confinement phase [4]. A structure like this is also what is seen by low-lying fermion modes [2,3]. The instanton liquid model [5] (built with calorons, at $T \neq 0$ ) seems to describe the background fields as superpositions of semiclassical objects. Confinement survives controlled smearing [4]. Therefore it is a challenge to describe the string tension in a gas or liquid model. With a liquid of instantons one fails to generate a linear potential between fundamental charges at $R>>\rho$ (instanton radius), and the static forces of different representations cannot be simultaneosly described, even at shorter distances.

After caloron solutions with non-trivial asymptotic holonomy have been discovered [6] the hope was revived to connect topology and confinement at the semiclassical level. Two things are required. First one has to find, below some temperature $T_{\mathrm{dec}}$, that the effective potential of the Polyakov loop, generated by a gas of (anti)calorons, develops a minimum at zero [7]. Second, one should be able to demonstrate that the caloron gas creates a non-vanishing (temporal) string tension, e.g. from the Polyakov loop correlator at finite temperature, if the asymptotic Polyakov loop is vanishing (confinement). The spatial string tension, derivable from spatial Wilson loops, should remain unaltered if the asymptotic Polyakov loop gets non-vanishing (deconfinement), and it should be rising at high temperature.

We will present preliminary results supporting such a scenario in the second part of this report. Before that, we will update our recent search for $\mathrm{KvB}$ caloron-like features [8] in Monte Carlo configurations analyzed after smearing. This type of analysis has been extended now to various temperatures below $T_{d e c}$.

\section{SU(2) Calorons at high and low temperature}

The distinguishing feature of the $\mathrm{KvB}$ caloron [6] is the existence of $N_{\text {color }}$ monopole constituents. Let $d$ be the distance between the two, for SU(2). The size of the constituent lumps is $\mathscr{O}(1 / \pi T)$. They share the action and topological charge in a proportion $\omega /\left(\frac{1}{2}-\omega\right)$ determined by the asymptotic holonomy, i.e. the Polyakov loop $\left.L_{\infty}=\cos (2 \pi \omega)\right)$. If the constituents are wellseparated relative to their size they form static lumps of action and charge. They are localizable by degenerate eigenvalues of the local holonomy, meaning $L(\vec{x}) \rightarrow \pm 1$ for SU(2) (see Fig. 1). In the maximal Abelian gauge (MAG), Abelian monopoles are emerging from Abelian projection that can serve to localize the (otherwise non-Abelian and gauge independent !) monopoles. Being sources of magnetic and electric fields $\left(\vec{B}^{a}= \pm \vec{E}^{a}\right)$, they are called dyons. At low temperature, a parameter $\rho$ (with $\rho^{2}=d /(\pi T)$ ) plays the role of the size of a single (instanton-like) lump.

The elusive fractional topological charge constituents [9] have also been seen using overimproved cooling on the symmetric torus, appearing as parts of a classical solution, with a size $\mathscr{O}(L)$ determined solely by the volume. In Monte Carlo (equilibrium) configurations such lumps get more localized as the result of quantum fluctuations. Such instanton constituents might have been 

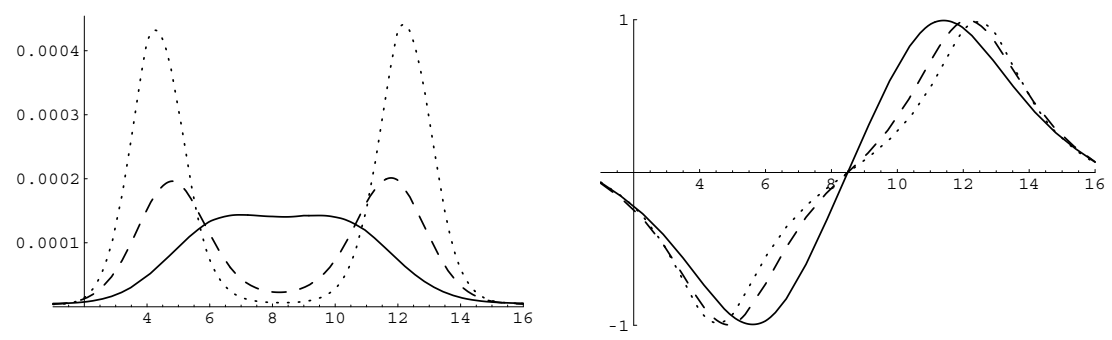

Figure 1: Action density (left) and Polyakov loop (right) along the axis between constituents at fixed distance in a SU(2) caloron for different temperatures. At lower $T$ the lumps coalesce whereas the peaks of the Polyakov loop still localize the constituents. [9]

detected on the symmetric torus already with the help of fermionic zero modes [10] which jump under the change of boundary conditions (periodic/antiperiodic). This interpretation is under scrutiny now together with these authors.

\section{Caloron-like clusters ? [8]}

We have examined the Abelian monopole content of topological clusters which become visible in smeared configurations. The aim was to identify lumps of charge, which are likely to be "undissociated" calorons, and those which are isolated dyons (single monopoles accompanied by topological charge). This study has been performed on 500 configurations on a $20^{3} \times 6$ lattice created at (Wilson) $\beta=2.3$. The configurations were subjected to $50 \ldots 100$ steps of $4 \mathrm{D}$ APEsmearing. A cluster analysis was then searching for connected clusters of lattice points with charge density $|q(x)|>q_{\text {threshold }}$. We have adapted the threshold to give a maximal number of separate clusters.

The distribution of the local Polyakov loop at sites passed by timelike Abelian monopoles was found very different from the unbiased local distribution. Then we considered topological clusters according to the monopoles detected inside. We should mention that many topological clusters remain unclassified in this way. The monopole lines occur either as isolated static monopoles, as monopole-antimonopole $(M \bar{M})$ pairs or as closed monopole trajectories. On this basis we have classified the clusters containing monopoles. We were mainly interested in two cluster observables. One is the averaged Polyakov loop $\langle P L \text { (Abelian monopoles) }\rangle_{\text {cluster (averaged over sites near time- }}$ like monopoles inside the clusters). In clusters with $M \bar{M}$ or with closed monopole trajectories this average turns out close to zero, for isolated monopoles the distribution of this observable is peaked far from zero (around \pm 0.75 ). The other cluster observable is the cluster topological charge $Q_{\text {cluster }}$, assigned to the cluster by summing up the topological density within a radius estimated from the maximal density in the cluster. This estimate is model dependent, taking the type of cluster into account. For details we refer to Ref. [8].

As a test, the method has been applied to single calorons randomly created with some distribution of distances $d$ between the constituents. The resulting scatterplot is shown in Fig. 2 (left). The clustering towards $\left(Q_{\text {cluster }},\langle P L(\text { Abelian monopoles })\rangle_{\text {cluster }}\right)=( \pm 1,0)$ and $\left( \pm \frac{1}{2}, \pm 1\right)$ reaffirms the method and the usefulness of the cluster variables. The same scatterplot for smeared equilibrium 

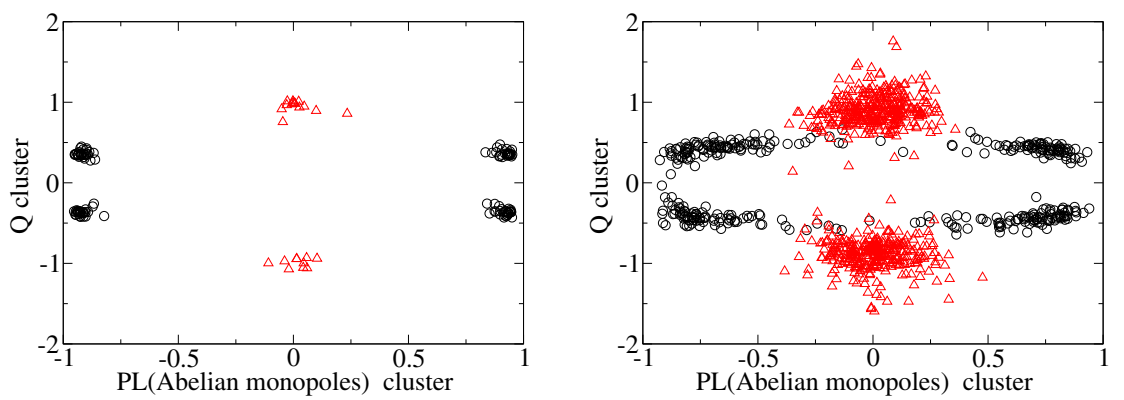

Figure 2: Scatterplots of topological clusters forming calorons (left) and in smeared Monte Carlo configurations (right) showing dyons (black circles) and non-dissociated calorons (red triangles).

configurations is shown in Fig. 2 (right). Both figures refer to MAG as the method to find the monopoles which are crucial for this analysis. We have also used the Polyakov gauge.
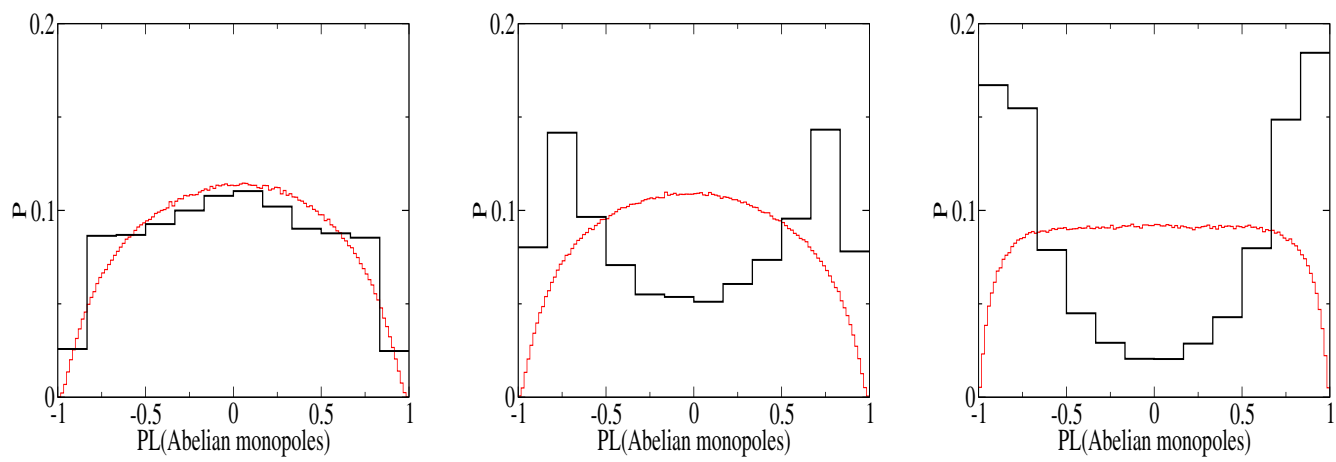

Figure 3: Distribution of Polyakov loops in sites with time-like Abelian monopoles (black thick line). For comparison the unbiased distribution of Polyakov loops in all sites is shown (red thin line). The subpanels show this for $\beta=2.2,2.3,2.4$ (from left to right).

Recently we have repeated this analysis with 200 configurations at (Wilson) $\beta=2.2,2.3$ and 2.4 (corresponding to $T / T_{\mathrm{dec}}=0.5,0.63$, and 0.88 ) in the confined phase. The lattice size was now $24^{3} \times 6$, and 50 steps of 4D APE-smearing have been applied. The MAG fixing has been refined (using now simulated annealing from 5 random start copies). Now we are in the position to see how the above picture depends on the temperature. As Fig. 3 shows, the correlation between Abelian monopoles and the Polyakov loop becomes stronger (weaker) with increasing (decreasing) $\beta$.

We have checked the contingency of the cluster classification according to monopoles in MAG and Polyakov gauge. Fig. 4 refers now only to clusters which are identically recognized in both gauges. The clustering in these scatterplots should be considered as a hint for the existence of calorons and dyons. It is obvious that the probability to detect the dyon gas increases towards $T_{\mathrm{dec}}$. At lower temperature we expect no evidence for separate dyons. At low $T$ topological clusters tagged by their monopole content are mostly non-static lumps with a charge clustering around \pm 1 . We are still unable, applying this method to the deconfined phase, to understand the topological objects there in terms of $\mathrm{KvB}$ calorons. 

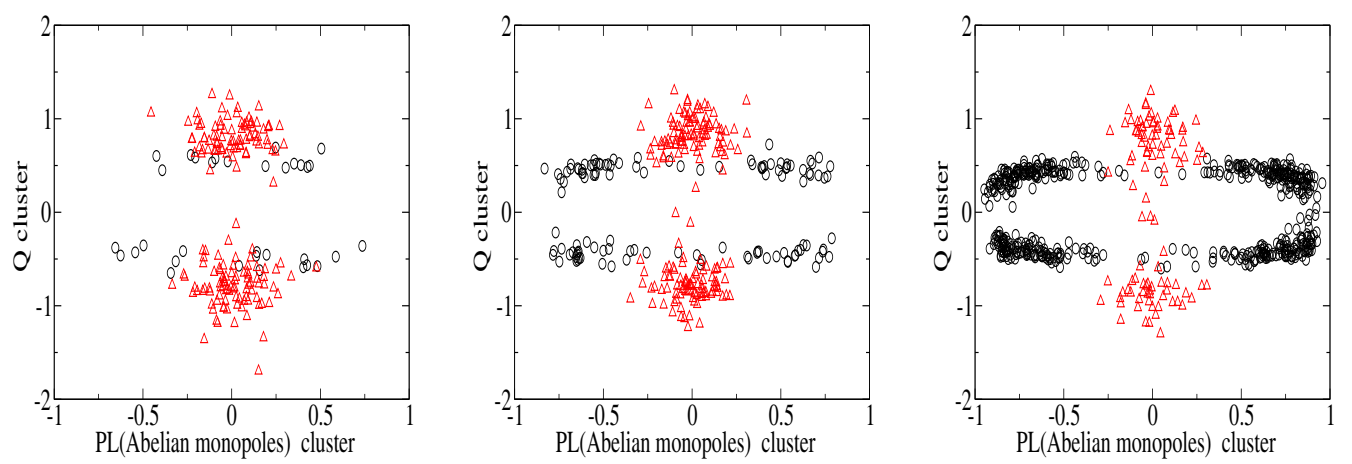

Figure 4: The Figure shows similar scatterplots as Fig. 2 b, now for $\beta=2.2,2.3,2.4$ (from left to right) in the refined analysis (see text).

\section{A SU(2) KvB caloron gas model}

We have constructed a model based on random superpositions of KvB calorons. There are technical details that can be described only in a forthcoming publication: fixed holonomy boundary conditions, how to avoid interaction of calorons to be added with Dirac strings of existing calorons, improving overlapping calorons. We consider an equal number of calorons and anticalorons randomly distributed in a continuous box. On an embedded open $32^{3} \times 8$ lattice the gauge field is discretized for further analysis. The temperature (and lattice scale) was chosen setting $T=T_{\mathrm{dec}}$, the deconfinement temperature. With a density $n=1 \mathrm{fm}^{-4}$, on average 64 (anti)calorons will occupy the lattice 4-volume. The aim is to demonstrate, in this conservative setting, the crucial role of the holonomy parameter $\omega$. Temperature $T$ and holonomy $\omega$ entering the $\mathrm{KvB}$ caloron solutions are prescribed whereas the constituent positions $x_{1}$ and $x_{2}$ are random (one of the time coordinates being equivalent to a global rotation). The 3D distance $d=\left|\vec{x}_{1}-\vec{x}_{2}\right|$ has been sampled according to a distribution obtained from $n(\rho) \propto \rho^{b-5} \exp \left(-c \rho^{2}\right), b=11 N_{\text {color }} / 3$ to give an average $\bar{\rho}=0.4$ $\mathrm{fm}$. This choice was suggested in order to remain close to the instanton model. It turns out that in this case only a small fraction of calorons is really dissociated into dyons.
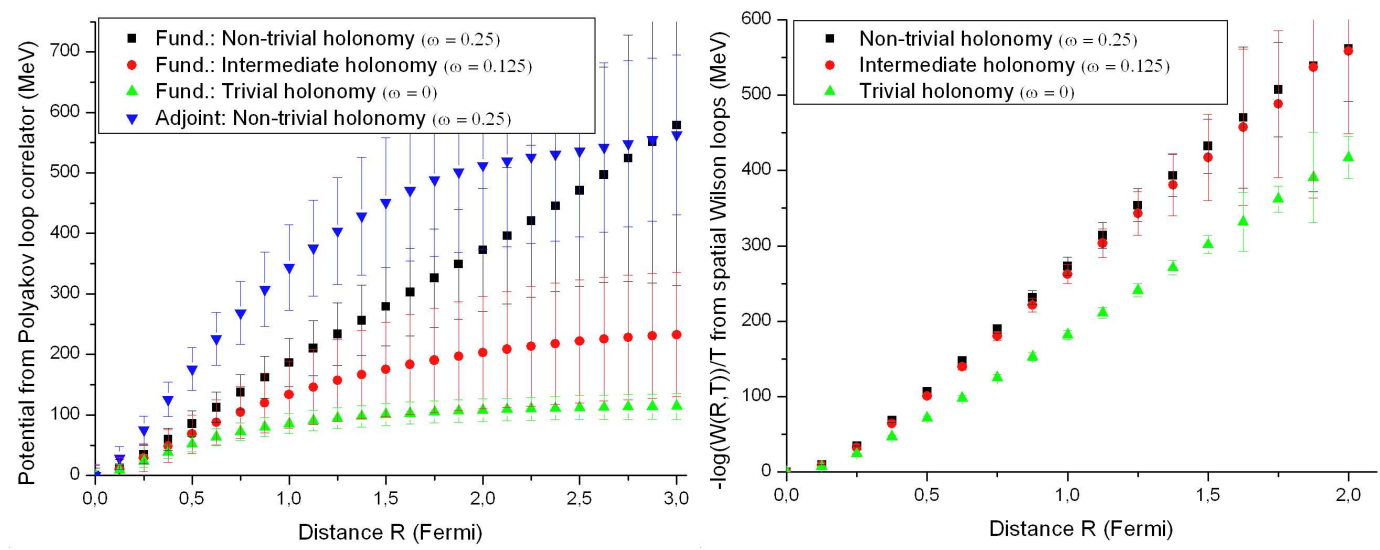

Figure 5: Left: the potential for fundamental and adjoint static charges from the corresponding Polyakov loop correlators; right: linearly rising "potential" for fundamental charges from spatial Wilson loops. Note the various asymptotic holonomies $\omega$. 
Fig. 5 (left) shows that the static potential of fundamental charges (shown as function of $R$ ) is strongly influenced by $\omega=1 / 4,1 / 8$ and 0 . Even for maximal non-trivial holonomy we observe string breaking for adjoint charges, whereas the initial rise of both potential corresponds to approximate Casimir scaling. Fig. 5 (right) presents $-\log W(R, T) / T$ from spatial Wilson loops demonstrating the existence of a spatial string tension even for trivial holonomy.

So far, the temperature dependence of the holonomy $L_{\infty}$ and of the density $n_{\text {cal }}$ was beyond the scope of our discussion. The complete model and its practical implementation (with realistic input distributions) will be described in the full paper.

\section{Summary}

We have updated our analysis to confirm KvB caloron-like correlations in smeared equilibrium configurations at various temperatures. We reported on progress constructing a dyon-caloron gas model comprising holonomy, confinement and deconfinement in the vicinity of $T_{\mathrm{dec}}$.

\section{Acknowledgments}

This work was supported by DFG (FOR 465 "Lattice Hadron Phenomenology". The ITEPHU collaboration took benefit from grants RFBR 03-02-16941, RFBR 04-02-16079 and DFG 436 RUS 113/739/0. E.-M. I. thanks for the hospitality in the group of P. van Baal at Leiden.

\section{References}

[1] I. Horvath et al., Local chirality of low lying Dirac eigenmodes and the instanton liquid model, Phys. Rev. D66 (2002) 034501 [hep-lat/ 0201008 ].

[2] Y. Koma et al., Localization properties of the topological charge density and the low lying eigenmodes of overlap fermions, PoS(LAT2005)300 (these proceedings).

[3] V. Weinberg et al., Probing the chiral phase transition of $N_{f}=2$ clover fermions with valence overlap fermions, PoS(LAT2005)171 (these proceedings).

[4] T. DeGrand, A. Hasenfratz, and T. G. Kovacs, Revealing topological structure in the SU(2) vacuum, Nucl. Phys. B520 (1998) 301-322 [hep-lat/9711032].

[5] E. V. Shuryak and T. Schäfer, Instantons in QCD, Rev. Mod. Phys. 70 (1998) 323-426 [hep-lat/9610451].

[6] T. C. Kraan and P. van Baal, Periodic instantons with non-trivial holonomy, Nucl. Phys. B533 (1998) 627-659, [hep-th/9805168].

[7] D. Diakonov, Instantons at work, Prog. Part. Nucl. Phys. 51 (2003) 173-222 [hep-ph/0212 026].

[8] E.-M. Ilgenfritz, B. V. Martemyanov, M. Müller-Preussker, A. I. Veselov, The monopole content of topological clusters: have KvB calorons been found?, Phys. Rev. D71 (2005) 034505 [hep-lat/0412028].

[9] F. Bruckmann, E.-M. Ilgenfritz, B. V. Martemyanov, P. van Baal, Probing for instanton constituents with $\varepsilon$-cooling, Phys. Rev. D70 (2004) 105013 [hep-lat/ 0408004 ].

[10] C. Gattringer and R. Pullirsch, Topological lumps and Dirac zero modes in SU(3) lattice gauge theory on the torus, Phys. Rev. D69 (2004) 094510 [hep-lat/ 0402008 ]; C. Gattringer and S. Solbrig, Dependence of Dirac eigenmodes on boundary conditions for SU(2) lattice gauge theory, [hep-lat/0410040]; 